NIKHEF/02-004

\title{
Cosmic scalar fields with flat potential*
}

\author{
J.W. van Holten \\ NIKHEF, Amsterdam NL**
}

June 23, 2002

\begin{abstract}
The dynamics of cosmic scalar fields with flat potential is studied. Their contribution to the expansion rate of the universe is analyzed, and their behaviour in a simple model of phase transitions is discussed.
\end{abstract}

\footnotetext{
${ }^{*}$ Work supported by the research program FP52 of the Foundation for Fundamental Research of Matter (FOM).

** e-mail: v.holten@nikhef.nl
} 

The universe is observed to expand [1], and this expansion is suspected to have accelerated at a late stage of its evolution [2, 3]. Furthermore, observation of the cosmic microwave background indicates the universe is spatially flat [4]. The energy density of scalar fields has been recognized to contribute to the expansion of the universe [5, 6, 6], and has been proposed to explain inflation [8], as well as the presently observed expansion [9, 10, 11, 12, 13]; in ref. [14] an attempt has been made to confront the data with the predictions for a minimally coupled scalar field with an a priori unknown potential. An overview of models with many references has been given in 15.

As suggested by the standard model of the electroweak interactions and its many proposed small-distance extensions with or without supersymmetry, the dynamics of the universe involves a large number of scalar fields interacting with gravity, gauge fields and fermionic matter. The observed isotropy and homogeneity of the universe do not allow for the existence of long-range electric and magnetic fields, but neutral scalar fields can have non-trivial dynamics in an expanding FRW-type universe.

For a number of minimally coupled scalar fields $\phi_{i}$ with a potential $V[\phi]$ the relevant dynamical equations for a flat universe $(k=0)$ are

$$
\ddot{\phi}_{i}+3 H \dot{\phi}_{i}+V_{, i}=0, \quad \frac{1}{2} \sum_{i} \dot{\phi}_{i}^{2}+V[\phi]+\rho=\frac{3 H^{2}}{8 \pi G},
$$

where $H$ is the Hubble parameter, and $\rho$ is the contribution of matter to the density of the universe. It should be noted, that we absorb any cosmological constant in a constant contribution to the potential $V[\phi]$.

Constant scalar fields. As observed in [5, 6, 7] simple non-trivial solutions to the coupled equations (11) are provided by constant fields at stationary points of the potential:

$$
V_{, i}=0, \quad V_{m}+\rho=\frac{3 H^{2}}{8 \pi G} .
$$

Here $V_{m}$ is the value of the potential at the stationary point (presumably a minimum), and $\rho$ represents the density of ordinary relativistic and cold nonrelativistic matter, with present densities $\rho_{r, 0}$ and $\rho_{n r, 0}$. Then the last equation becomes in terms of the scale factor $\sigma(t)=a(t) / a_{0}$ :

$$
\dot{\sigma}^{2}=h^{2} \sigma^{2}+\frac{8 \pi G}{3}\left(\frac{\rho_{r, 0}}{\sigma^{2}}+\frac{\rho_{n r, 0}}{\sigma}\right), \quad h^{2}=\frac{8 \pi G V_{m}}{3},
$$

provided $V_{m}>0$. Hence for large universes the late-time behaviour of the scale factor is described by

$$
a(t) \sim a_{0} e^{h\left(t-t_{0}\right)} .
$$

This is consistent with the observed present state of the universe if the Hubble parameter is $h \simeq 70 \mathrm{~km} / \mathrm{sec} / \mathrm{Mpc}$, of the order of the inverse life time of the universe. As is well-known, the corresponding value of $V_{m}$ is extremely small: $V_{m}=\rho_{c} \simeq 10^{-122}$ in Planck units.

In contrast, for $V_{m}=0$, we obtain the standard radiation/matter dominated solutions with $a(t) \sim t^{\kappa}$, where $\kappa=1 / 2$ or $\kappa=2 / 3$. If $V_{m}=-3 \omega^{2} /(8 \pi G)<0$, 
there are oscillating solutions with maximal amplitude $\bar{a}=\bar{\sigma} a_{0}$ given by the solution of

$$
\omega^{2} \bar{\sigma}^{4}-\frac{8 \pi G}{3}\left(\rho_{n r, 0} \bar{\sigma}+\rho_{r, 0}\right)=0 .
$$

The last two cases can be consistent with a flat universe only if the density of matter and radiation equals or exceeds the critical density $\rho_{c}$, which is definitely contradicted by the best current estimates of the densities of luminous and cold dark matter.

Flat potentials. A different evolution of the Hubble parameter $H(t)$ is obtained by allowing one or more scalar fields to depend on time. We consider the situation where the first field is dynamical: $\phi_{1}=\varphi(t)$, whilst all other fields are constant: $\phi_{i}=v_{i}(i=2,3, \ldots, N)$. Then $V(\varphi)=V\left[\phi_{1}=\varphi ; \phi_{i}=v_{i}\right]$. Thus we have

$$
\ddot{\varphi}+3 H \dot{\varphi}+V^{\prime}=0, \quad \frac{1}{2} \dot{\varphi}^{2}+V(\varphi)+\rho=\frac{3 H^{2}}{8 \pi G} .
$$

In general any solution depends sensitively on the initial value $\varphi_{\text {init }}$, which is difficult to control. However, this problem is absent if the potential is invariant under field translations $\tilde{\varphi}=\varphi+\epsilon$. Therefore it is of some interest to study flat potentials $V(\varphi)=V_{0}$. Such potentials are characteristic for Goldstone bosons, and they occur in many supersymmetric models. From the flatness of $V$ one derives a conservation law for the field momentum per co-moving volume:

$$
\ddot{\varphi}+3 H \dot{\varphi}=0 \quad \Rightarrow \quad \sigma^{3} \dot{\varphi}=\gamma=\text { constant }
$$

After multiplication by $\sigma^{6}$ the other equation then becomes

$$
\frac{\gamma^{2}}{2}+\rho \sigma^{6}+V_{0} \sigma^{6}=\frac{3}{8 \pi G}\left(\sigma^{3} H\right)^{2}=\frac{1}{24 \pi G}\left(\frac{d \sigma^{3}}{d t}\right)^{2}
$$

The solutions can be characterized qualitatively as in the case of constant scalar fields:

a. For $V_{0}>0$, the potential provides the leading term, and for large $a$ we find as before:

$$
a(t) \simeq a_{0} e^{h\left(t-t_{0}\right)}, \quad h=\sqrt{\frac{8 \pi G V_{0}}{3}} .
$$

b. For $V_{0}=0$, the leading term is provided by the matter density $\rho$, and the evolution of the scale factor is again of the form $a(t) \sim t^{\kappa}$, where $\kappa=1 / 2$ for relativistic matter, and $\kappa=2 / 3$ for cold non-relativistic matter.

c. Finally, for $V_{0}<0$ there are oscillating solutions, with the maximal scale $\bar{a}=\bar{\sigma} a_{0}$ a solution of

$$
\frac{\gamma^{2}}{2}+\bar{\rho} \bar{\sigma}^{6}+V_{0} \bar{\sigma}^{6}=0
$$

which is a direct generalization of eq.(可).

Matter-dominated regime. The precise evolution of the scale factor and the scalar field can be solved analytically in the matter-dominated regime, in which $\rho a^{3}=$ constant. In the following we describe the solutions in some detail. 
It is convenient to use the dimensionless scale variable $\sigma=a / a_{0}=1 /(1+z)$. Assuming $V_{0} \neq 0$, eq.(8) then becomes

$$
\left(\frac{d \sigma^{3}}{d t}\right)^{2}=24 \pi G V_{0}\left[\left(\sigma^{3}+\frac{\rho_{0}}{2 V_{0}}\right)^{2}+\frac{\gamma^{2}}{2 V_{0}}-\frac{\rho_{0}^{2}}{4 V_{0}^{2}}\right],
$$

where $\rho(\sigma)=\rho_{0} / \sigma^{3}$. As this equation is invariant under time reversal, at any time we can have an increasing and a decreasing solution; we always exhibit here the solution with growing scale factor at earely times. For positive values of $V_{0}$ we must distinguish three cases:

a. $\gamma^{2}>\rho_{0}^{2} / 2 V_{0}>0$. Then the solution of eq.(11) is

$$
\sigma(t)=\frac{a(t)}{a_{0}}=\left[A \sinh 3 h\left(t-t_{0}-\Delta\right)-\frac{\rho_{0}}{2 V_{0}}\right]^{1 / 3},
$$

with

$$
A=\sqrt{\frac{\gamma^{2}}{2 V_{0}}-\frac{\rho_{0}^{2}}{4 V_{0}^{2}}}, \quad 3 h \Delta=-\operatorname{arcsinh}\left[\frac{1}{A}\left(1+\frac{\rho_{0}}{2 V_{0}}\right)\right],
$$

and $h$ given in eq.(9). The corresponding solution for the scalar field is

$$
\varphi(\sigma)=\varphi_{0}-\frac{1}{\sqrt{12 \pi G}}\left(\operatorname{arcsinh}\left[\frac{1}{\sigma^{3}} \frac{\gamma^{2}+\rho_{0} \sigma^{3}}{\sqrt{2 \gamma^{2} V_{0}-\rho_{0}^{2}}}\right]-\Omega\right),
$$

with the intergration constant given by

$$
\Omega=\operatorname{arcsinh}\left[\frac{\gamma^{2}+\rho_{0}}{\sqrt{2 \gamma^{2} V_{0}-\rho_{0}^{2}}}\right] .
$$

As all equations are invariant under reflections of the scalar field: $\varphi \rightarrow-\varphi$, there is another solution

$$
\varphi(\sigma)=\varphi_{0}+\frac{1}{\sqrt{12 \pi G}}\left(\operatorname{arcsinh}\left[\frac{1}{\sigma^{3}} \frac{\gamma^{2}+\rho_{0} \sigma^{3}}{\sqrt{2 \gamma^{2} V_{0}-\rho_{0}^{2}}}\right]-\Omega\right) .
$$

b. $\gamma^{2}=\rho_{0}^{2} / 2 V_{0}$. In this case the constant terms in the square brackets in (11) cancel, with the result that

$$
\sigma(t)=\left[1+\left(1+\frac{\rho_{0}}{2 V_{0}}\right)\left(e^{3 h\left(t-t_{0}\right)}-1\right)\right]^{1 / 3} .
$$

The solution for the scalar field reads

$$
\varphi(\sigma)=\varphi_{0} \mp \frac{1}{\sqrt{12 \pi G}} \ln \left[\frac{1}{\sigma^{3}} \frac{\gamma^{2}+\rho_{0} \sigma^{3}}{\gamma^{2}+\rho_{0}}\right] .
$$

To obtain the coefficients in front of and inside the logarithm, we have used the relation between $V_{0}$ and $h$, and the special relation between $V_{0}$ and $\rho_{0}$ and $\gamma$. c. $\gamma^{2}<\rho_{0}^{2} / 2 V_{0}$. The solution of eq.(11) becomes

$$
\sigma(t)=\left[A \cosh \left[3 h\left(t-t_{0}-\Delta\right)\right]-\frac{\rho_{0}}{2 V_{0}}\right]^{1 / 3}
$$


with

$$
A=\sqrt{\frac{\rho_{0}^{2}}{4 V_{0}^{2}}-\frac{\gamma^{2}}{2 V_{0}}}, \quad 3 h \Delta=\operatorname{arccosh}\left[\frac{1}{A}\left(1+\frac{\rho_{0}}{2 V_{0}}\right)\right] .
$$

The scalar field evolution is given by

$$
\varphi(\sigma)=\varphi_{0} \mp \frac{1}{\sqrt{12 \pi G}}\left(\operatorname{arccosh}\left[\frac{1}{\sigma^{3}} \frac{\gamma^{2}+\rho_{0} \sigma^{3}}{\sqrt{\rho_{0}^{2}-2 V_{0} \gamma^{2}}}\right]-\Omega\right),
$$

where the constant $\Omega$ is

$$
\Omega=\operatorname{arccosh}\left[\frac{\gamma^{2}+\rho_{0}}{\sqrt{\rho_{0}^{2}-2 V_{0} \gamma^{2}}}\right] .
$$

Observe, that in all three solutions above $\varphi$ diverges for $\sigma \rightarrow 0$, as does $\dot{\varphi}$. However, long before this limit is reached the universe becomes relativistic, and our model must be modified. The initial conditions for the model discussed here should rather be set at the onset of the matter-dominated aera.

d. If $V_{0}=0$ equation (8) implies

$$
\sigma(t)=\left[1+\sqrt{12 \pi G\left(2 \rho_{0}+\gamma^{2}\right)}\left(t-t_{0}\right)+6 \pi G \rho_{0}\left(t-t_{0}\right)^{2}\right]^{1 / 3} .
$$

(We have chosen the solution which can vanish at $t=0$.) This is the usual cold matter dominated universe. The scalar field equation is most easily solved directly in terms of time:

$$
\varphi(t)=\varphi_{0} \mp \frac{1}{\sqrt{3 \pi G}}\left(\operatorname{arctanh}\left[\frac{\sqrt{12 \pi G} \rho_{0}\left(t-t_{0}+\Delta\right)}{\gamma}\right]-\Omega\right),
$$

with

$$
\Delta=\sqrt{\frac{\gamma^{2}+2 \rho_{0}}{12 \pi G \rho_{0}^{2}}}, \quad \Omega=\operatorname{arctanh} \sqrt{1+\frac{2 \rho_{0}}{\gamma^{2}}} .
$$

This solution is asymptotically constant;

$$
\varphi_{\infty}=\varphi_{0} \pm \frac{1}{\sqrt{3 \pi G}}\left(\operatorname{arctanh}\left[\sqrt{1+\frac{2 \rho_{0}}{\gamma^{2}}}\right]-1\right) .
$$

The field can have reached its asymptotic value at present only if $\gamma^{2} \ll \rho_{0}$. e. For $V_{0}<0$, the scale factor takes the periodic form

$$
\sigma(t)=\left[\frac{\rho_{0}}{2\left|V_{0}\right|}+A \sin 3 \omega\left(t-t_{0}-\Delta\right)\right]^{1 / 3}
$$

with

$$
A=\sqrt{\frac{\rho_{0}^{2}}{4 V_{0}^{2}}+\frac{\gamma^{2}}{2\left|V_{0}\right|}}, \quad \omega=\sqrt{\frac{8 \pi G\left|V_{0}\right|}{3}}
$$


and

$$
3 \omega \Delta=\arcsin \left[\frac{1}{A}\left(\frac{\rho_{0}}{2\left|V_{0}\right|}-1\right)\right] .
$$

The scalar field solution reads

$$
\varphi(\sigma)=\varphi_{0} \mp \frac{1}{\sqrt{12 \pi G}}\left(\operatorname{arccosh}\left[\frac{1}{\sigma^{3}} \frac{\gamma^{2}+\rho_{0} \sigma^{3}}{\sqrt{2\left|V_{0}\right| \gamma^{2}+\rho_{0}^{2}}}\right]-\Omega\right),
$$

where

$$
\Omega=\operatorname{arccosh}\left[\frac{\gamma^{2}+\rho_{0}}{\sqrt{2\left|V_{0}\right| \gamma^{2}+\rho_{0}^{2}}}\right]
$$

Actually, this is formally the same solution as for the scalar field in case (c). However, it evolves in a universe with a maximum expansion and a finite life time, rather than an infinitely expanding one.

Equation of state. As cold matter is pressureless, the total pressure of the cosmic fluid comes from the scalar field:

$$
p_{t o t}=p_{s}=\frac{\gamma^{2}}{2 \sigma^{6}}-V_{0}
$$

The total density is

$$
\rho_{t o t}=\rho_{s}+\rho=\frac{\gamma^{2}}{2 \sigma^{6}}+\frac{\rho_{0}}{\sigma^{3}}+V_{0}
$$

It follows that the state parameter is given by

$$
w_{t o t}=\frac{p_{t o t}}{\rho_{t o t}}=-\frac{2 V_{0} \sigma^{6}-\gamma^{2}}{2 V_{0} \sigma^{6}+2 \rho_{0} \sigma^{3}+\gamma^{2}} .
$$

For infinitely expanding universes with $V_{0}>0$, in the large-scale limit the parameter becomes $w_{\infty}=-1$. Inflationary behaviour sets in when $w_{\text {tot }}<-1 / 3$. This happens at a critical scale $\sigma_{e}$ given by

$$
\sigma_{e}^{3}=\frac{\rho_{0}}{4 V_{0}}\left(1+\sqrt{1+\frac{16 \gamma^{2} V_{0}}{\rho_{0}^{2}}}\right) .
$$

Depending on the relative values of $V_{0}$ and $\rho_{0}^{2} / 2 \gamma^{2}$, we have

$$
\begin{aligned}
& \gamma^{2}>\rho_{0}^{2} / 2 V_{0} \quad \rightarrow \quad \sigma_{e}^{3}>\frac{\rho_{0}}{V_{0}} \\
& \gamma^{2}=\rho_{0}^{2} / 2 V_{0} \quad \rightarrow \quad \sigma_{e}^{3}=\frac{\rho_{0}}{V_{0}} \\
& \gamma^{2}<\rho_{0}^{2} / 2 V_{0} \rightarrow \sigma_{e}^{3}<\frac{\rho_{0}}{V_{0}} .
\end{aligned}
$$

For vanishing potential $V_{0}=0$ the parameter is always positive, but approaches $w_{\text {tot }} \downarrow 0$ in the large-scale limit. For $V_{0}<0$ the state parameter $w_{t o t}$ is always positive and becomes infinite at the turning point $\sigma=\bar{\sigma}$. 
The ratio of dark energy to cold matter is given by

$$
y=\frac{\rho_{s}}{\rho}=\frac{\gamma^{2}}{2 \rho_{0}} \frac{1}{\sigma^{3}}+\frac{V_{0}}{\rho_{0}} \sigma^{3} .
$$

For $V_{0}>0$ it never becomes small, but it reaches a minimum for $\sigma_{m}^{6}=\gamma^{2} / 2 V_{0}$, when

$$
y_{m}=\sqrt{\frac{2 V_{0} \gamma^{2}}{\rho_{0}^{2}}} .
$$

Thus a situation in which $y_{m}<1$ can be reached only if $\gamma^{2}<\rho_{0}^{2} / 2 V_{0}$. In this case

$$
\frac{\sigma_{e}^{3}}{\sigma_{m}^{3}}<\frac{2}{y_{m}}
$$

From this analysis we observe, that a recent acceleration of the universe conjectured on the basis of the type Ia supernova data can be incorporated in these simple models for $V_{0}>0$. By construction the development of the scalar field does not depend strongly on the initial conditions, as in more sophisticated models with tracking behaviour [16]. Also, we can arrange for the scalar density to be comparatively low in the early universe, and have accelerated expansion at a later time if we take the parameters in the domain $0<\gamma^{2}<\rho_{0}^{2} / 2 V_{0}$.

Piece-wise constant potentials. The analysis of flat potentials can be extended in a straightforward way to the case of piece-wise constant potentials. The main new feature is that whenever the field reaches a potential step, we must determine and match its behaviour before and after it hits the discontinuity. This is done by rewriting the field equation in the form

$$
\frac{d}{d t}\left(\sigma^{3} \dot{\varphi}\right)=-\sigma^{3} V^{\prime} \Rightarrow \frac{d}{d t}\left(\frac{1}{2}\left(\sigma^{3} \dot{\varphi}\right)^{2}\right)=-\sigma^{6} \frac{d V}{d t} .
$$

The second equation can be integrated under two different conditions:

(i) if the field crosses the potential step (transmission), we obtain

$$
\frac{\Delta \gamma^{2}}{2 \bar{\sigma}^{6}}=-\Delta V
$$

with $\Delta V=V_{2}-V_{1}$ the magnitude of the potential step, $\bar{\sigma}$ the scale at which the field reaches the discontinuity in the potential, and $\Delta \gamma^{2}=\gamma_{2}^{2}-\gamma_{1}^{2}$ the change in the square of the field momentum;

(ii) if the field does not cross the potential step (reflection), the value of the potential does not change, and $\gamma^{2}$ is equal before and after the reflection. As the field now must run in the other direction as a function of time, we deduce that $\gamma_{2}=-\gamma_{1}$.

The condition (41) can also be written in the form

$$
\frac{\gamma_{1}^{2}}{2 \bar{\sigma}^{6}}+V_{1}=\frac{\gamma_{2}^{2}}{2 \bar{\sigma}^{6}}+V_{2}
$$

As matter is not created nor destroyed, this shows, that $H$ is continuous during the change in the scalar field. In contrast, during transmission across the potential step the derivative of the field $\dot{\varphi}$, the derivative of the Hubble parameter 
$\dot{H}$ and the potential all change by a finite amount. This can also be derived by taking a derivative of the second eq.(6), which leads to the equations

$$
-\frac{\dot{H}}{8 \pi G}=\frac{1}{2} \dot{\varphi}^{2}+\frac{1}{2} \rho, \quad \frac{3 H^{2}+\dot{H}}{8 \pi G}=V+\frac{1}{2} \rho .
$$

From the continuity of $\rho$ and $H$, and the finite discontinuity of $V$, the finite discontinuous changes in $\dot{H}$ and $\dot{\varphi}$ are obtained directly:

$$
\Delta V=\frac{\Delta \dot{H}}{8 \pi G}=-\Delta\left(\frac{1}{2} \dot{\varphi}^{2}\right)=-\frac{\Delta\left(\gamma^{2}\right)}{2 \bar{\sigma}^{6}} .
$$

The same equations actually also hold trivially at reflection, as all changes in these quantities then vanish. In this case continuity of the field is obtained by taking a solution $\varphi(\sigma)=\varphi_{1}(\sigma)$ before the reflection $(\sigma<\bar{\sigma})$, and $\varphi(\sigma)=\varphi_{2}(\sigma)$ after the reflection $(\sigma>\bar{\sigma})$, with the two solutions related by

$$
\varphi_{2}(\sigma)=2 \bar{\varphi}-\varphi_{1}(\sigma), \quad \bar{\varphi}=\varphi_{1}(\bar{\sigma})=\varphi_{2}(\bar{\sigma}) .
$$

Observe, that if the initial condition is such that

$$
\frac{\gamma_{1}^{2}}{2 \bar{\sigma}^{6}}+V_{1}<V_{2}
$$

then only the reflected solution exists.

Obviously, the piece-wise constant potentials discussed here can be used as approximations for a variety of smoothly changing potentials. It is to be noted that the translation symmetry in field space is then lost, but one still has relative freedom in choosing initial conditions in any flat region of the potential.

Phase transitions. In the course of evolution of the universe, phase transitions accompanied by symmetry breaking can change the value of the potential. We consider the simple situation that the potential changes from a value $V_{1}$ before the transition time $t_{c}$ to a value $V_{2}$ after $t_{c}$. For simplicity we take the transition to occur instantaneously. We can then treat the phase transition as a potential step occurring at the time $t_{c}$. Eq. (42) then implies, that during the transition

$$
\frac{\gamma_{1}^{2}}{2 \sigma_{c}^{6}}+V_{1}=\frac{\gamma_{2}^{2}}{2 \sigma_{c}^{6}}+V_{2}
$$

where $\sigma_{c}=\sigma\left(t_{c}\right)$. Note, that in this case we only have the possibility of transmission, not reflection of the field. As the matter density and Hubble parameter are not affected during the transition, the continuity of $\sigma(t)$ and $\varphi(t)$ allow us to express the parameters of the solution for $t>t_{c}$ in terms of those of the solution for $t<t_{c}$.

In this context, consider again the parameter $y_{m}=\sqrt{2 \gamma^{2} V / \rho_{0}^{2}}$, the minimal ratio of scalar to matter density. From eq.(47) it follows, that after a phase transition

$$
y_{m 2}^{2}-y_{m 1}^{2}=\frac{2 \sigma_{c}^{6}}{\rho_{0}^{2}}\left(V_{1}^{2}-V_{2}^{2}\right)+\frac{1}{2 \rho_{0}^{2} \sigma_{c}^{6}}\left(\gamma_{1}^{4}-\gamma_{2}^{4}\right) \text {. }
$$


Thus, whether the transition leads to a situation with larger or smaller $y_{m}$ depends both on the difference in potential energy density as well as on the difference in kinetic energy density of the field. In particular, the value remains the same if

$$
\frac{V_{1}}{V_{2}}=\frac{\gamma_{2}^{2}}{\gamma_{1}^{2}} \quad \Rightarrow \quad \frac{\gamma_{1}^{2}}{2 \sigma_{c}^{6}}=V_{2}, \quad \frac{\gamma_{2}^{2}}{2 \sigma_{c}^{6}}=V_{1}
$$

i.e. if the values of kinetic and potential energy are interchanged during the transition. In all cases where this condition is not met, the value of $y_{m}$ changes. If the potential energy density dominates over the kinetic energy density, than lowering $V$ will in general lower $y_{m}$, whereas in kinetic-energy dominated situations a lowering of $V$ will give rise to a larger value of $y_{m}$ after the transition. This follows from the observation, that eq.(48) can be replaced by the equivalent expression

$$
y_{m 2}^{2}-y_{m 1}^{2}=\frac{2 \sigma_{c}^{6}}{\rho_{0}^{2}}\left(V_{1}-V_{2}\right)\left(V_{1}+V_{2}-\frac{\gamma_{1}^{2}+\gamma_{2}^{2}}{2 \sigma_{c}^{6}}\right) .
$$

As generically the nature of the solution for $\sigma(t)$ and $\varphi(t)$ depends on whether $y_{m}^{2}$ is positive, zero or negative, and in the first case on whether it is equal to, larger or less than unity, the solutions before and after a phase transition may belong to different classes a-e discussed above. It should be noted that the analysis also applies to the case where an originally constant field $\left(\gamma_{1}=0\right)$ becomes dynamical $\left(\gamma_{2} \neq 0\right)$, in which case $V_{1}=V_{2}+\gamma_{2}^{2} / 2 \sigma_{c}^{6}$.

In conclusion, massless scalar fields associated with flat potentials display simple but interesting cosmological dynamics. As they are associated with Goldstone bosons, they can play a role in phase transitions during which they change their dynamics. Finally, it has been shown how to piece together the solutions for potentials with discrete jumps, which under certain conditions can give qualitative insight in the behaviour of non-constant potentials.

\section{References}

[1] E. Hubble, Proc. Nat. Acad. Sci. 15 (1929), 168

[2] S. Perlmutter et al., Nature 391 (1998), 51

[3] A.G. Riess et al., Astr. J. 116 (1998), 1009

[4] A.H. Jaffe et al., Phys. Rev. Lett. 86 (2001), 3475

[5] A. Linde, JETP Lett. 19 (1974), 183

[6] J. Dreitlein, Phys. Rev. Lett. 33 (1974), 1243

[7] M. Veltman, Cosmology and the Higgs mechanism (preprint may 1974, unpublished)

[8] A. Guth, Phys. Rev. D23 (1981), 347

[9] C. Wetterich, Nucl. Phys. B302 (1988), 668 
[10] P.J.E. Peebles and B. Ratra, Ap. J. Lett. 325 (1988), L17

[11] R.R. Caldwell, R. Dave and P.J. Steinhardt, Phys. Rev. Lett. 80 (1998), 1582

[12] C. Armendariz-Picon, V. Mukhanov and P.J. Steinhardt, Phys. Rev. Lett. 85 (2000), 4438

[13] A. Hebecker and C. Wetterich, Phys. Lett. B497 (2001), 281

[14] T.D. Saini, S. Raychaudhury, V. Shani and A.A. Starobinsky, Phys. Rev. Lett. 85 (2000), 1162

[15] V. Sahni, arXiv:astro-ph/0202076

[16] I. Zlatev, L. Wang and P.J. Steinhardt, Phys. Rev. Lett. 82 (1999), 896 